\title{
Production of Induced Secondary Metabolites by a Co-Culture of Sponge-Associated Actinomycetes, Actinokineospora sp. EG49 and Nocardiopsis sp. RV163
}

\section{Yousef Dashti ${ }^{1}$, Tanja Grkovic ${ }^{1}$, Usama Ramadan Abdelmohsen ${ }^{2, \dagger}$, Ute Hentschel ${ }^{2}$ and Ronald J. Quinn ${ }^{1, *}$}

1 Eskitis Institute for Drug Discovery, Griffith University, Brisbane, QLD 4111, Australia;

E-Mails: yousef.dashti@griffithuni.edu.au (Y.D.); t.grkovic@griffith.edu.au (T.G.)

2 Department of Botany II, Julius-von-Sachs Institute for Biological Sciences, University of Würzburg, Julius-von-Sachs-Platz 3, D-97082 Würzburg, Germany;

E-Mails: usama.ramadan@uni-wuerzburg.de (U.R.A.); ute.hentschel@uni-wuerzburg.de (U.H.)

$\dagger$ Permanent address: Department of Pharmacognosy, Faculty of Pharmacy, Minia University, Minia 61519, Egypt

* Author to whom correspondence should be addressed; E-Mail: r.quinn@griffith.edu.au; Tel.: +61-7-3735-6006; Fax: +61-7-3735-6001.

Received: 7 January 2014; in revised form: 4 March 2014 / Accepted: 10 April 2014 /

Published: 22 May 2014

\begin{abstract}
Two sponge-derived actinomycetes, Actinokineospora sp. EG49 and Nocardiopsis sp. RV163, were grown in co-culture and the presence of induced metabolites monitored by ${ }^{1} \mathrm{H}$ NMR. Ten known compounds, including angucycline, diketopiperazine and $\beta$-carboline derivatives 1-10, were isolated from the EtOAc extracts of Actinokineospora sp. EG49 and Nocardiopsis sp. RV163. Co-cultivation of Actinokineospora sp. EG49 and Nocardiopsis sp. RV163 induced the biosynthesis of three natural products that were not detected in the single culture of either microorganism, namely $\quad N$-(2-hydroxyphenyl)-acetamide (11), 1,6-dihydroxyphenazine (12) and 5a,6,11a,12-tetrahydro-5a,11a-dimethyl[1,4]benzoxazino[3,2-b][1,4]benzoxazine (13a). When tested for biological activity against a range of bacteria and parasites, only the phenazine 12 was active against Bacillus sp. P25, Trypanosoma brucei and interestingly, against Actinokineospora sp. EG49. These findings highlight the co-cultivation approach as an effective strategy to access the bioactive secondary metabolites hidden in the genomes of marine actinomycetes.
\end{abstract}


Keywords: co-cultivation; induced metabolites; sponge-associated actinomycetes; NMR fingerprint; bioactivity

\section{Introduction}

The search for novel biologically active natural products sourced from marine microbes continues to be an important endeavour fuelled by the emergence of new infections diseases and chemotherapy resistance. Marine-derived actinomycete collections have recently yielded new compounds with not only potent biological activity, but also novel molecular scaffolds, for example salinosporamide A [1] and marinopyrroles A and B [2]. Salinosporamide A was shown to be an irreversible inhibitor of the $20 \mathrm{~S}$ proteasome and entered clinical trials against multiple myeloma, only three years after its discovery [3]. However, finding new microbial secondary metabolites is becoming difficult, as the rate of rediscovery of known compounds is increasing [4,5]. On the other hand, genomic sequencing has revealed the presence of a large number of putative biosynthetic gene clusters in the genomes of some microorganisms that encode for secondary metabolites that are not seen under classical cultivation conditions [6-8]. Different strategies have been proposed to activate these cryptic biosynthetic pathways. Co-fermentation of microorganisms in a single environment is one of the proposed methods to de-silence biosynthetic pathways for the production of new secondary metabolites [7,9-11]. Mixed fermentation of two or more microbes can make a competitive environment, which may induce unexpressed pathways and result in the synthesis of bioactive secondary metabolites due to interspecies crosstalk or chemical defence mechanisms [11-13].

Examples of the production of induced new natural products by mixed fermentation of marine-sourced microorganisms include a chlorinated benzophenone pestalone [14] sourced from Pestalotia sp. strain CNL-365 and marine $\alpha$-proteobacterium strain CNJ-328, the diterpenoids libertellenones A-D isolated from a co-culture of the same bacterial strain CNJ-328 with the fungus, Libertella sp. CNL-52 [15], and cyclic depsipeptides emericellamides A and B isolated from a co-culture of marine-derived fungus Emericella sp. (CNL-878) and marine bacterium Salinispora arenicola [16]. In this work, we focus on the induced metabolites from the co-cultivation of two sponge-sourced actinomycetes. Several in-house strains were co-cultured and the presence of differential secondary metabolite production monitored by UV-Vis, MS and NMR techniques. Two strains, namely Actinokineospora sp. and Nocardiopsis sp., when grown in co-culture showed different chemical profiles to that of the mono-cultures and were prioritised for large-scale natural product isolation work.

Members of the genus Actinokineospora were isolated from soil, plants [17,18] and marine sponges [19]. Although this genus is not well known for secondary metabolite production, we recently reported two new angucycline-like compounds named actinosporins A (1) and B (2) from Actinokineospora sp. EG49, where actinosporin A displayed anti-parasitic activity against Trypanosoma brucei brucei [20]. On the other hand, the genus, Nocardiopsis, is frequently isolated from terrestrial, as well as marine environments, including marine sponges [21-23]. Members of this genus are prolific producers of a multitude of secondary metabolites with diverse activities [24-27]. 
In this study, two sponge-derived actinomycetes were co-cultured in liquid media; these being Nocardiopsis sp. RV163 from the Mediterranean sponge Dysidea avara, and Actinokineospora sp. EG49 from the Red Sea sponge, Spheciospongia vagabunda. To the best of our knowledge, this is the first report of induced metabolites from the mixed fermentation of two sponge-associated actinomycetes.

Traditionally, the detection of induced metabolite biosynthesis has relied either on LC-PDA [5,28,29] or LC-PDA-MS [12,15,16,30], methods to monitor the production of the secondary metabolite by comparison of the small molecule profiles of the mono- and co-cultures of microorganisms. However, these analytical techniques are dependent either on the existence of a chromophore (PDA detection) or the ability of a compound to be ionised (MS detection) and might not detect all of the changes of the secondary metabolome between the mono- and co-cultures. In order to further interrogate the existence of the induced change in the secondary metabolome profiles, we used LC-PDA, as well as ${ }^{1} \mathrm{H}-\mathrm{NMR}$ fingerprinting techniques. Following the detection of the production of the induced metabolites, an isolation process was performed, which led to the identification of $\mathrm{N}$-(2-hydroxyphenyl)-acetamide (11), 1,6-dihydroxyphenazine (12) and 5a,6,11a,12-tetrahydro-5a,11a-dimethyl[1,4]benzoxazino[3, 2-b][1,4]benzoxazine (13a).

\section{Results and Discussion}

The mono- and co-culture secondary metabolite profiles were monitored with a combination of UV-PDA and NMR-based spectroscopic techniques. Figure 1 depicts the LC-PDA metabolic profile of the three actinomycetes cultures and shows that the co-culture extract displayed a very different chemotype compared to that of the two single cultures. In order to further investigate the differences of the secondary metabolite profiles by ${ }^{1} \mathrm{H}-\mathrm{NMR}$ and to have a sufficient quantity to identify the metabolites, a large-scale study was undertaken on $50 \mathrm{mg}$ of the EtOAc extract. The methodology utilised identical reversed-phase $\mathrm{C}_{18}$ stationary support as for the analytical HPLC run, but allowed for a longer elution gradient, which gave better sensitivity and resolution of the secondary metabolites present. ${ }^{1} \mathrm{H}-\mathrm{NMR}$ spectra were then used to compare the differences between each chromatography fraction sourced from the mono- and co-cultures.

\subsection{Monoculture Chemical Profiles}

Previously, we reported on the structures of two new angucycline-type metabolites, actinosporins A (1) and B (2) (Figure 2), isolated from Actinokineospora sp. EG49 [20]. In this work, compounds 1 and 2 were confirmed to be the major metabolites present in this extract, and further attempts at the structure elucidation of minor metabolites were not made. The majority of the natural products present in the EtOAc extract of Nocardiopsis sp. RV163 belonged to the diketopiperazine class of compounds. They were identified as 2,5-diketopiperazines cyclo-(prolyl-valyl) (3) [31], cyclo-(isoleucyl-prolyl) (4) [32], cyclo-(leucyl-prolyl) (5) [31], cyclo-(prolyl-tyrosyl) (6) [33], cyclo-(phenylalanyl-prolyl) (7) [32] and cyclo-(prolyl-tryptophyl) (8) $[34,35]$. The purity of the compounds (at $<90 \%$ ) prevented us from confirming the absolute configuration of the diketopiperazines, 3-8. Two other secondary metabolites in Nocardiopsis sp. RV163 EtOAc extract were identified as known natural products 1-hydroxy-4-methoxy-2-naphthoic acid (9) [36] and 1-acetyl- $\beta$-carboline (10) [37]. 
Figure 1. HPLC chromatograms of the EtOAc extracts of Actinokineospora sp. EG49 and Nocardiopsis sp. RV163 co-culture (top), Actinokineospora sp. EG49 monoculture (middle) and Nocardiopsis sp. RV163 monoculture (bottom). The depicted chromatograms were extracted at 210,254, 280 and $380 \mathrm{~nm}$, and the bottom two spectra show representative examples of the natural products isolated from the two strains.

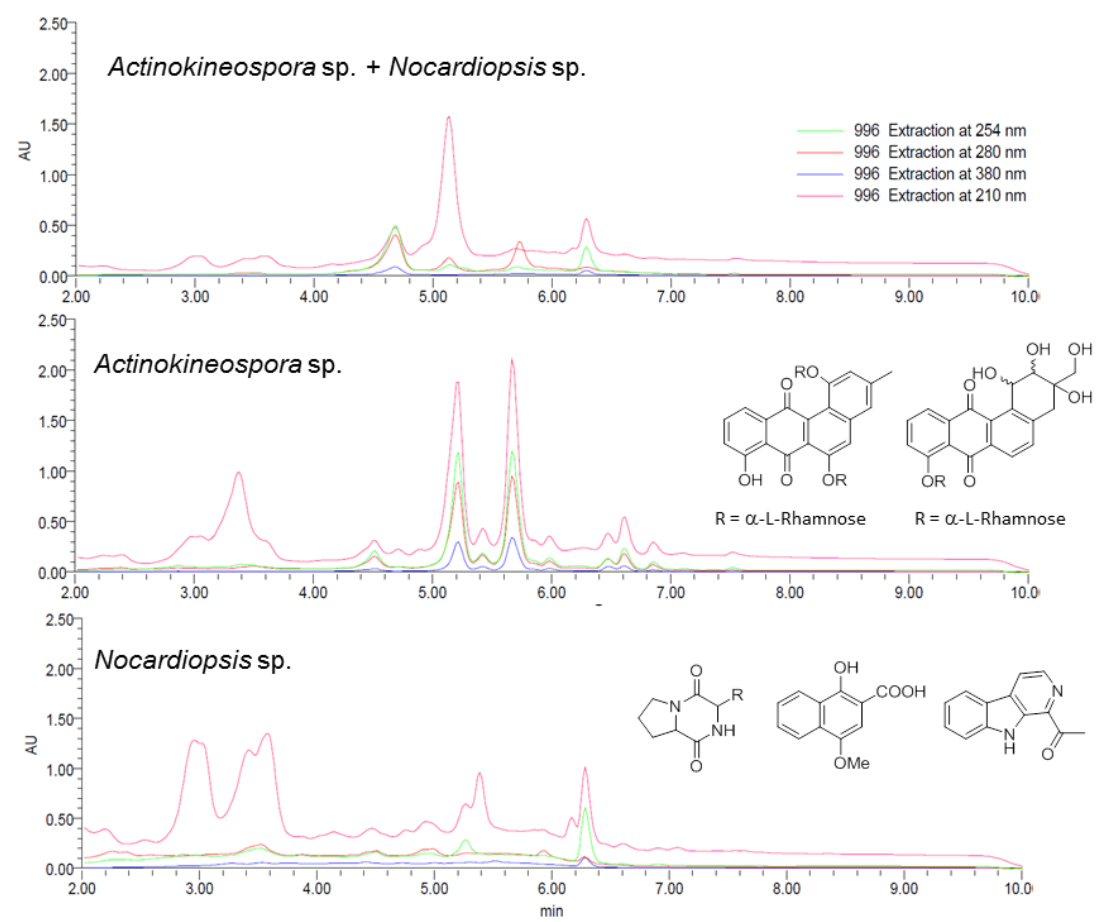

Figure 2. The structure of the major compounds identified from the EtOAc extracts of Actinokineospora sp. EG49 actinosporins A (1) and B (2); Nocardiopsis sp. RV163 cyclo-(prolyl-valyl) (3), cyclo-(isoleucyl-prolyl) (4), cyclo-(leucyl-prolyl) (5), cyclo-(prolyl-tyrosyl) (6), cyclo-(phenylalanyl-prolyl) (7), cyclo-(prolyl-tryptophyl) (8), 1-hydroxy-4-methoxy-2-naphthoic acid (9) and 1-acetyl- $\beta$-carboline (10); and the co-culture, $\quad N$-(2-hydroxyphenyl)-acetamide (11), 1,6-dihydroxyphenazine (12), 5a,6,11a,12-tetrahydro-5a,11a-dimethyl-1,4-benzoxazino[3,2-b][1,4]benzoxazine and 2,2',3,3'-tetrahydro-2,2'-dimethyl-2,2'-bibenzoxazole (13b).
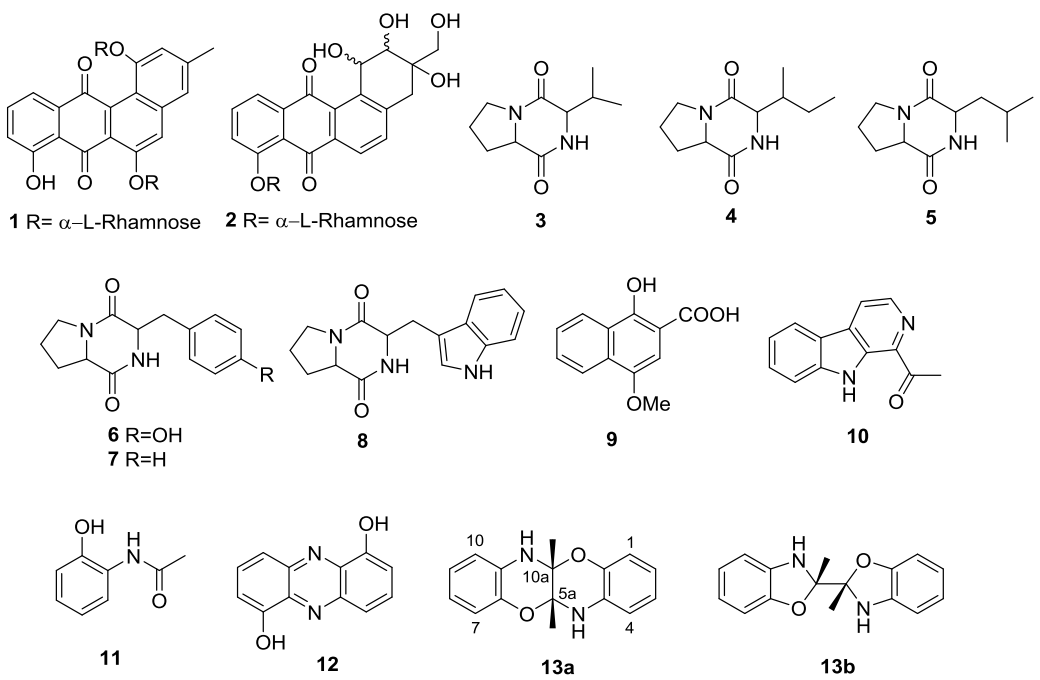


\subsection{Co-Culture Chemical Profile}

Having established the UV-PDA, MS and the ${ }^{1} \mathrm{H}-\mathrm{NMR}$ profile of the two monocultures, the co-culture extract was investigated. The ${ }^{1} \mathrm{H}-\mathrm{NMR}$ spectra of the same chromatography fractions of mono- and co-culture extracts were compared, and since the retention times of compounds can vary, the neighbouring fractions were also considered. The presence of the first induced metabolite was apparent in the ${ }^{1} \mathrm{H}$-NMR spectra of fraction 5 of co-culture compared to that of the mono-cultures. Aromatic signals in the region 6.70 to $7.70 \mathrm{ppm}$ were observed in co-culture that were not present in the spectra of the Actinokineospora sp. EG49- and Nocardiopsis sp. RV163-sourced fractions (Figure 3a). The absence of these NMR signals in both monocultures suggested that this was an induced metabolite produced through mixed fermentation of the two actinomycetes. A literature search based on the molecular ion and structural information generated from the ${ }^{1} \mathrm{H}-\mathrm{NMR}$ spectrum identified this compound to be the known natural product, $N$-(2-hydroxyphenyl)-acetamide (11) [38].

Figure 3. ${ }^{1} \mathrm{H}-\mathrm{NMR}$ fingerprints of HPLC fractions sourced from the EtOAc extracts of Actinokineospora sp. EG49 and Nocardiopsis sp. RV163 co-culture (top in black), Actinokineospora sp. EG49 monoculture (middle in blue) and Nocardiopsis sp. RV163 monoculture (bottom in red). (a) ${ }^{1} \mathrm{H}-\mathrm{NMR}$ spectra of fraction 5; chemical shifts for the induced metabolite $\mathrm{N}$-(2-hydroxyphenyl)-acetamide (11) are highlighted; (b) ${ }^{1} \mathrm{H}-\mathrm{NMR}$ fingerprints of fraction 24; the possible presence of compound $\mathbf{1 2}$ in the Actinokineospora sp. EG49 monoculture is highlighted.

\section{a}

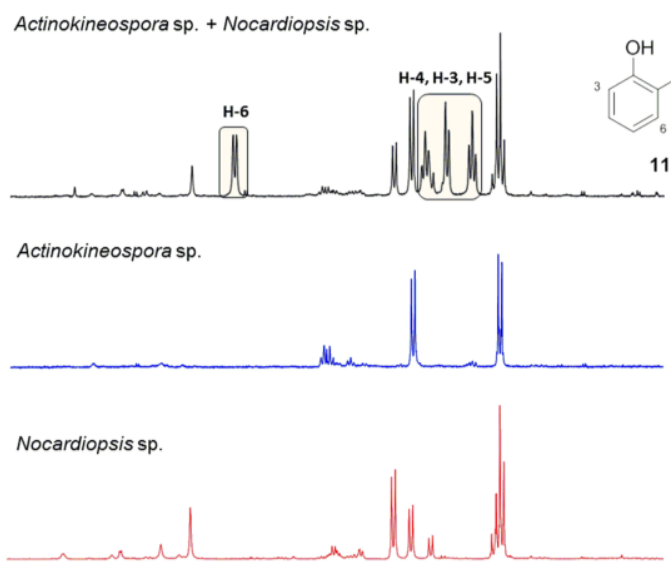

b

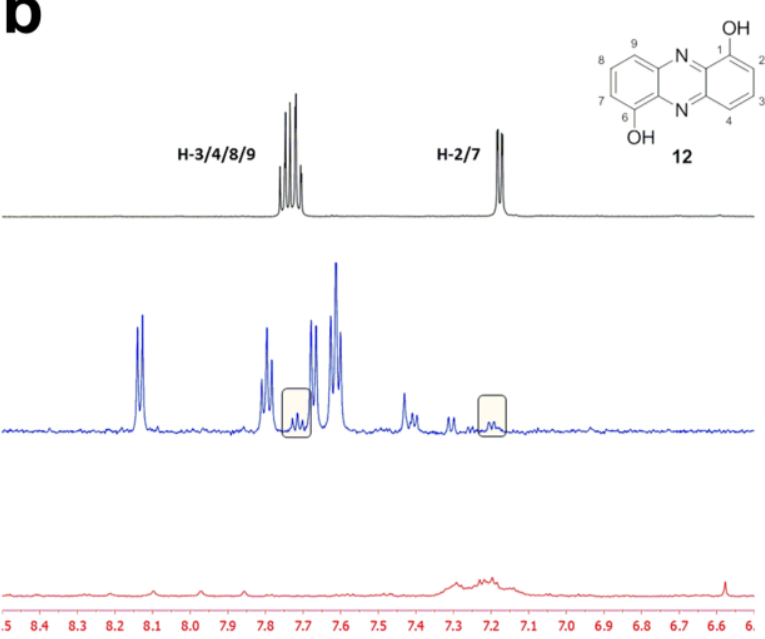

In addition to fraction 5, two other chromatography fractions showed the presence of induced metabolites. In regions concentrated around fraction 24, aromatic signals between 7.15 and 7.80 ppm and an exchangeable one at $10.45 \mathrm{ppm}$ were detected in co-culture, and while initially this compound was not apparent in either of the two monocultures, the ${ }^{1} \mathrm{H}-\mathrm{NMR}$ fingerprint suggested that this metabolite may be present in very small amounts in the Actinokineospora sp. EG 49 extract (Figure 3b). In order to further interrogate whether compound 12 was present in the Actinokineospora $\mathrm{sp}$. EG49 monoculture extract, we performed an NMR titration experiment (see Supplementary Information). NMR-detected titration of pure 12 into fraction 24 sourced from the Actinokineospora sp. EG49 extract 
demonstrated that compound $\mathbf{1 2}$ was not present in the mono-culture sample and confirmed that this small molecule is another induced metabolite from the mixed fermentation experiment. Based on the comparison of the mass and the NMR information with the literature, the induced metabolite was identified to be 1,6-dihydroxyphenazine (12) [39]. Compound 12 was produced in a very high yield ( $12 \%$ crude weight), and being in such a high abundance we found that this major component was present in the NMR fingerprints of many of the consequent fractions of the co-culture extract. Fractions 26 and 27, in addition to having ${ }^{1} \mathrm{H}-\mathrm{NMR}$ signals of 1,6-dihydroxyphenazine, showed additional proton signals in the aromatic region, which were not present in either of the monoculture extracts (for the NMR fingerprint comparison, see Supplementary Information). The spectroscopic and spectrometric data of the third induced metabolite were consistent with the structures of 5a,6,11a,12-tetrahydro[1,4]benzoxazino[3,2-b][1,4]benzoxazine (13a) and 2,2',3,3'-tetrahydro-2, 2'-dimethyl-2,2'-bibenzoxazole (13b) (Figure 2). Both compounds have previously been reported as the condensation product of 2-propyn-1-ol and dibenzo-2-amino alcohol, with the cis-fused benzoxazino-benzoxazine structure, 13a, confirmed as the correct product via single crystal X-ray analysis [40]. In this work, an insufficient amount of the compound has been isolated to provide a good quality crystal for an X-ray, and the assignment was based on NMR data. However, in contrast to $\left[2,2^{\prime}\right]$ bifuranyl-pyranopyran models $[41]$, the ${ }^{13} \mathrm{C}$ chemical shifts of the ring fusion atoms in 13a and 13b do not provide the distinction of the ring size [42]. Based on the literature evidence of compound 13a being the major isomer in solution [43], we propose the structure of the induced natural product to be 5a,6,11a,12-tetrahydro-5a,11a-dimethyl[1,4]benzoxazino[3,2-b][1,4]benzoxazine (13a). Since this is the first report of compound 13a as a natural product, a complete set of NMR data is given in pyridine- $d_{5}$.

In addition to tracking the production of the induced metabolites in the co-culture extract, the NMR experiment was also useful in showing the suppression of the production of some natural products. For example, while in Figure 3b, the main compound in fraction 24 sourced from the Actinokineospora sp. EG49 crude extract is actinosporin B (2) (blue, middle spectrum), in the co-culture NMR spectrum (black, top spectrum), all resonances for compound $\mathbf{2}$ are missing, indicating that the mixed fermentation suppressed the production of actinosporin B. In support of the NMR observations, the mixed culture of the two microbes was dominated by the Nocardiopsis sp. RV163, which is characterized by large brown colonies and the production of spores, while very little growth was observed by the yellow cells of the Actinokineospora sp. EG49.

\subsection{Anti-Infective Activity of Induced Metabolites}

The three induced compounds, $N$-(2-hydroxyphenyl)-acetamide (11), 1,6-dihydroxyphenazine (12) and 5a,6,11a,12-tetrahydro-5a,11a-dimethyl[1,4]benzoxazino[3,2-b][1,4]benzoxazine (13a), were tested for their activities against Bacillus sp. P25, Escherichia coli and Fusarium sp. P21, human parasites Leishmania major and Trypanosoma brucei, as well as Nocardiopsis sp. RV163 and Actinokineospora sp. EG49 cultures. Biological activity was documented for compound $\mathbf{1 2}$ against Bacillus sp. (11 mm inhibition zone diameter), Trypanosoma brucei ( $\mathrm{IC}_{50}$ value of $\left.19 \mu \mathrm{M}\right)$ and, interestingly, against Actinokineospora sp. EG49 (15 $\mathrm{mm}$ inhibition zone diameter) (see Supplementary Information). 


\section{Experimental Section}

\subsection{General Experimental Procedures}

Optical rotations were measured on a JASCO P-1020 polarimeter with a $10-\mathrm{cm}$ cell. UV spectra were acquired on a Jasco V650 UV/vis spectrophotometer. A Jasco J-715 spectropolarimeter was used to record circular dichroism spectra. NMR spectra were recorded at $30{ }^{\circ} \mathrm{C}$ on a Varian Inova $600 \mathrm{MHz}$ spectrometer equipped with a triple resonance 5-mm cold probe. For NMR fingerprint experiments, the samples were dissolved in $230 \mu \mathrm{L}$ of DMSO- $d_{6}$ and run in a 3-mm NMR tube. The standard VnmrJ 3.2 Proton pulse sequence was run with the following parameters: $\mathrm{pw}=45^{\circ}, \mathrm{p} 1=0 \mu \mathrm{s}, \mathrm{d} 2=0 \mathrm{~s}, \mathrm{~d} 1=1 \mathrm{~s}$, at $=1.7 \mathrm{~s}, \mathrm{sw}=9615 \mathrm{~Hz}, \mathrm{nt}=8$ scans. LC-MS spectra were obtained using a Waters ZQ electrospray mass spectrometer with a Phenomenex Luna $\mathrm{C}_{18}$ column $(4.6 \mathrm{~mm} \times 50 \mathrm{~mm}, 3 \mu \mathrm{m})$ (Phenomenex, Torrance, CA, USA). Analytical HPLC was done with a Phenomenex Onyx Monolithic $\mathrm{C}_{18}$ column $(4.6 \times 100 \mathrm{~mm})\left(\right.$ Phenomenex, Torrance, CA, USA). A Phenomenex Onyx Monolithic $\mathrm{C}_{18}$ column $(10 \mathrm{~mm} \times 100 \mathrm{~mm})$ (Phenomenex, Torrance, CA, USA) was used for semi-preparative HPLC separation. All HPLC and LC-MS experiments were performed with a $\mathrm{MeOH}-\mathrm{H}_{2} \mathrm{O}$ gradient solvent system. Millipore Milli-Q PF filtered $\mathrm{H}_{2} \mathrm{O}$ and HPLC grade solvents were used for chromatography.

\subsection{Microbial Fermentation and Extracts Preparation}

Nocardiopsis sp. RV163 was isolated from the Mediterranean sponge, Dysidea avara, while Actinokineospora sp. EG49 was cultivated from the Red Sea sponge, Spheciospongia vagabunda [19]. Each strain was fermented in 8 Erlenmeyer flasks $(2 \mathrm{~L})$, each containing $1 \mathrm{~L}$ of ISP 2 (International Streptomyces Project) medium in artificial sea water and incubated at $30{ }^{\circ} \mathrm{C}$ for 7 days with shaking at $150 \mathrm{rpm}$. For co-cultivation experiment, $10 \mathrm{~mL}$ of 5-day-old culture of Nocardiopsis sp. RV163 was inoculated into 8 Erlenmeyer flasks ( $2 \mathrm{~L}$ ), each containing $1 \mathrm{~L}$ of ISP 2 medium inoculated with $10 \mathrm{~mL}$ of 5-day-old culture of Actinokineospora sp. EG49. After fermentation of single cultures and co-culture, filtration was done, and the supernatant was extracted with ethyl acetate $(2 \times 500 \mathrm{~mL})$ to give the ethyl acetate extract. XAD16 resin was then added to the mother liquor, shaken, filtered and finally extracted with acetone (acetone extract). The cells and mycelia were macerated in a double volume of methanol with shaking for $3 \mathrm{~h}$, then filtered (methanolic extract).

\subsection{Extraction and Isolation}

\subsubsection{General Chromatographic Procedures for Large-Scale Isolation and Fingerprinting Work}

Crude extract (50 mg) was chromatographed using HPLC on a semi-preparative Phenomenex Onyx Monolithic reversed-phase $\mathrm{C}_{18}$ column $(10 \mathrm{~mm} \times 100 \mathrm{~mm})$. Initially, isocratic conditions of $10 \% \mathrm{MeOH}$ were used for $10 \mathrm{~min}$, then a linear gradient from $10 \%$ to $100 \% \mathrm{MeOH}$ was performed over $40 \mathrm{~min}$ and continued isocratically for $10 \mathrm{~min}$ at a flow rate of $9 \mathrm{~mL} / \mathrm{min}$. Sixty fractions collected in one minute increments over 60 min were dried for NMR and mass studies. 


\subsubsection{Actinokineospora sp. EG49}

EtOAc extract $\left(50 \mathrm{mg}\right.$ ) was pre-adsorbed to $\mathrm{C}_{18}$-bonded silica and then packed into a stainless steel HPLC guard cartridge $(10 \times 30 \mathrm{~mm})$ that was subsequently attached to a $\mathrm{C}_{18}$ HPLC column. Standard gradient conditions described above were employed to give actinosporin B (10) in fraction 24 and actinosporin A (1) in fraction 31.

\subsubsection{Nocardiopsis sp. RV163}

EtOAc extract $\left(50 \mathrm{mg}\right.$ ) was pre-adsorbed to $\mathrm{C}_{18}$-bonded silica and then packed into a stainless steel HPLC guard cartridge $(10 \times 30 \mathrm{~mm})$ that was subsequently attached to a $\mathrm{C}_{18}$ HPLC column. Standard gradient conditions described above were employed. Fraction 9 was a mixture of cyclo-(prolyl-valyl) (3), cyclo-(isoleucyl-prolyl) (4) and cyclo-(leucyl-prolyl) (5). Semi-pure cyclo-(prolyltyrosyl) (6) (1.6 mg) and cyclo-(phenylalanyl-prolyl) (7) (1.0 mg) were identified from fractions 15 and 16, respectively, cyclo (prolyl-tryptophyl) (8) was identified from fraction 17. 1-hydroxy-4-methoxy-2-naphthoic acid (9) $(0.7 \mathrm{mg})$ and 1-acetyl- $\beta$-carboline (10) $(0.2 \mathrm{mg})$ were identified in fractions 26,27 and 30, respectively.

\subsubsection{Actinokineospora sp. EG49 and Nocardiopsis sp. RV163 Co-Culture}

EtOAc extract $\left(50 \mathrm{mg}\right.$ ) was pre-adsorbed to $\mathrm{C}_{18}$-bonded silica and then packed into a stainless steel HPLC guard cartridge $(10 \times 30 \mathrm{~mm})$ that was subsequently attached to a $\mathrm{C}_{18}$ HPLC column. Standard gradient conditions described above were employed to give $N$-(2-hydroxyphenyl)-acetamide (11) (0.3 mg) purified from fraction 5; diketopiperazines (3-8) were eluted in the same fractions as the mono-culture extract of Nocardiopsis sp.; the major induced compound, 1,6-dihydroxyphenazine (12) (5.9 mg), started to elute in fraction 24 and continued to elute off the column until fraction 42 . Fractions 25 to 28 were combined and re-run on a reverse-phase HPLC using Phenomenex Onyx Monolithic $\mathrm{C}_{18}$ column $(10 \mathrm{~mm} \times 100 \mathrm{~mm})$ eluting with a gradient from $\mathrm{H}_{2} \mathrm{O} / \mathrm{MeOH}(80: 20$ to $40: 60$ over $60 \mathrm{~min})$ to purify compound 13a $(0.6 \mathrm{mg})$.

\subsection{Bioactivity Testing}

\subsubsection{Antibacterial Activity}

The induced compounds 11, 12 and 13a were tested for their antimicrobial activity using the standard disk diffusion assay against Bacillus sp. P25, Escherichia coli and Fusarium sp. P21, as well as the actinomycetes from where the compounds were derived, Nocardiopsis sp. RV163 and Actinokineospora sp. EG49. Sterile filter disks (6-mm diameter) loaded with the test compounds ( $25 \mu \mathrm{L}$ of $1 \mathrm{mg} / \mathrm{mL}$ in methanol) were placed on agar plates that had been inoculated with $100 \mu \mathrm{L}$ of the test microorganism (cultures with an optical density of $\left.\mathrm{OD}_{600}=0.2\right)$. After incubation $(24 \mathrm{~h}$ for Bacillus, Escherichia coli and Fusarium sp. and $72 \mathrm{~h}$ for Nocardiopsis sp. RV163 and Actinokineospora sp. EG49) at $37{ }^{\circ} \mathrm{C}$ (Bacillus, Escherichia coli) and $30{ }^{\circ} \mathrm{C}$ (Fusarium sp., Nocardiopsis sp. RV163 and Actinokineospora sp. EG49), the antimicrobial potential was quantitatively assessed as the diameter of the inhibition zone $(n=2)$. 


\subsubsection{Anti-Trypanosomal Activity}

Anti-trypanosomal activity was tested following the protocol of Huber and Koella [44]. In complete Baltz medium, $10^{4}$ trypanosomes per millilitre of Trypanosoma brucei strain TC 221 were cultivated. Trypanosomes were tested in 96-well plate chambers against different concentrations of test substances at $0.25-40 \mu \mathrm{M}$ in $1 \%$ DMSO to a final volume of $200 \mu \mathrm{L}$. For controls, $1 \%$ DMSO, as well as parasites without any test compounds were used simultaneously in each plate to show no effect of $1 \%$ DMSO. The plates were then incubated at $37{ }^{\circ} \mathrm{C}$ in an atmosphere of $5 \% \mathrm{CO}_{2}$ for $24 \mathrm{~h}$. After the addition of $20 \mu \mathrm{L}$ of Alamar Blue, the activity was measured after 48 and $72 \mathrm{~h}$ by light absorption using an MR 700 Microplate Reader at a wavelength of $550 \mathrm{~nm}$ with a reference wavelength of $650 \mathrm{~nm}$. The $\mathrm{IC}_{50}$ value effect of the test compound was quantified by the linear interpolation of three independent measurements.

The following equation was used to calculate $\mathrm{IC}_{50}$ :

$$
\log \left(\mathrm{IC}_{50}\right)=\log \left(\mathrm{X}_{1}\right)+\left\{\left[\left(\mathrm{Y}_{1}-0.5\right) /\left(\mathrm{Y}_{1}-\mathrm{Y}_{2}\right)\right] \times\left[\log \left(\mathrm{X}_{2}\right)-\log \left(\mathrm{X}_{1}\right)\right]\right\}
$$

$\mathrm{Y}_{1}$ mean of the duplicate determination of the first measured cell density that is less than half the average of the growth control divided by the average of control growth.

$\mathrm{X}_{1}$ concentration of the substance that belongs to the cell density of $\mathrm{Y}_{1}$.

$\mathrm{Y}_{2}$ mean of the duplicate determination of the first measured cell density that is greater than half the average of the growth control divided by the average of control growth.

$\mathrm{X}_{2}$ concentration of the substance that belongs to the cell density of $\mathrm{Y}_{2}$.

\subsubsection{Anti-Leishmanial Activity}

Anti-leishmanial activity was tested following the method of Ponte-Sucre et al. [45]. Briefly, $10^{7}$ cells $/ \mathrm{mL}$ Leishmania major promastigotes were incubated in complete medium for $24 \mathrm{~h}$ at $26{ }^{\circ} \mathrm{C}$, $5 \% \mathrm{CO}_{2}$ and $95 \%$ humidity in the absence or presence of different concentrations of the test compounds $(0.25-40 \mu \mathrm{M}$ in $1 \%$ DMSO) to a final volume of $200 \mu \mathrm{L}$. Following the addition of Alamar Blue, the plates were incubated again, and the optical densities were determined after $48 \mathrm{~h}$ with a Multiskan Ascent enzyme-linked immunosorbent assay (ELISA) reader (Multiskan Ascent, Germany). The effects of cell density, incubation time and the concentration of DMSO were examined in control experiments. The results were expressed in $\mathrm{IC}_{50}$ values by linear interpolation of three independent experiments.

\subsection{Structure Elucidation of Compounds 1-13}

The structures of all known compounds were confirmed upon comparison of spectrometric (low-resolution MS) and spectroscopic $\left({ }^{1} \mathrm{H},{ }^{13} \mathrm{C}\right.$ and $2 \mathrm{D}$, where necessary) data with that of the published literature values.

5a,6,11a,12-tetrahydro-5a,11a-dimethyl[1,4]benzoxazino[3,2-b][1,4]benzoxazine (13a): Yellow oil; $[\alpha]_{\mathrm{D}}^{25}=0(c 0.012, \mathrm{MeOH}) ; \mathrm{UV}(\mathrm{MeOH}) \lambda_{\max }(\log \varepsilon), 295(3.61), 235(3.77), 208(4.36) \mathrm{nm} ;{ }^{1} \mathrm{H},{ }^{13} \mathrm{C}$ NMR data in DMSO- $d_{6}$ in good agreement with published values; ${ }^{1} \mathrm{H}-\mathrm{NMR}$ (pyridine- $\left.d_{5}, 600 \mathrm{MHz}\right) \delta$ $(J$ in $\mathrm{Hz}) 1.75$ (s, 2CH$), 6.80$ (t, 7.6, H-3/9), 6.90 (t, 7.6, H-2/8), 6.98 (d, 2H, H-4/10), 7.03 (d, 2H, 
$\mathrm{H}-1 / 7), 8.00(\mathrm{~s}, 2 \mathrm{NH}) ;{ }^{13} \mathrm{C}$ NMR (pyridine- $\left.d_{5}, 125 \mathrm{MHz}\right) 22.2\left(2 \mathrm{CH}_{3}\right), 83.5(\mathrm{C}-5 \mathrm{a} / 11 \mathrm{a}), 115.5(\mathrm{C}-1 / 7)$, 117.6 (C-4/10), 120.2 (C-3/9), 121.9 (C-2/8), 131.8 (C-6a/12a), 143.5 (C-4a/10a), LRESIMS m/z $269.3[\mathrm{M}+\mathrm{H}]^{+}$.

\section{Conclusions}

The genomes of microorganisms, particularly of the order, Actinomycetales, consist of a large number of putative biosynthetic gene clusters that encode for secondary metabolites that are not produced using standard fermentation protocols. To reach this cryptic treasure trove of natural products, approaches, such as co-cultivation, are required to induce them. In this study, two sponge-derived actinomycetes, Nocardiopsis sp. RV163 and Actinokineospora sp. EG49, were co-fermented in liquid media. The presence of induced metabolites was studied by comparison of the ${ }^{1} \mathrm{H}-\mathrm{NMR}$ fingerprints of the crude extracts of the two monocultures and the co-culture. The NMR fingerprint allowed the confidence of detecting all small molecules containing proton nuclei and was instrumental in demonstrating that the induced metabolites were co-culture specific and not present in either of the single cultures. Co-cultivation of Nocardiopsis sp. RV163 and Actinokineospora sp. EG49 induced the biosynthesis of three compounds, which were not detected in either microorganism in a single culture, namely $N$-(2-hydroxyphenyl)-acetamide (11), 1,6-dihydroxyphenazine (12) and 5a,6,11a,12-tetrahydro-5a, 11a-dimethyl[1,4]benzoxazino[3,2-b][1,4]benzoxazine (13a). When tested for biological activity against a range of bacteria and parasites, only the phenazine, 12, was active against Bacillus sp. P25, Trypanosoma brucei and interestingly against Actinokineospora sp. EG49. Moreover, while not detectable by ${ }^{1} \mathrm{H}-\mathrm{NMR}$ in the monocultures, compound $\mathbf{1 2}$ was produced in a very high yield ( $12 \%$ crude weight) by the co-culture of the two microbes. These findings highlight the co-cultivation approach as an effective strategy to increase the yield of metabolites undetected in the single microbial culture and enhance the chemical diversity of the secondary metabolites hidden in the genomes of marine actinomycetes.

\section{Acknowledgments}

The authors wish to acknowledge funding from the Australian Research Council for support toward NMR and MS equipment (LE0668477 and LE0237908). We thank Heike Bruhn (Deutsche Forschungsgemeinschaft SFB 630 TP Z1) for the anti-infective assays. Financial support was provided by DFG (Sonderforschungsbereiche SFB 630 TP A5) to Ute Hentschel. We acknowledge Safwat Ahmed and Amro Hanora (Suez Canal University, Egypt) for collaborations.

\section{Author Contributions}

Y.D. preformed the natural product isolation work. Y.D and T.G. identified the structure of the natural products. U.R.A cultured the microorganisms and performed the biological assays. Y.D., T.G., U.R.A., U.H., R.J.Q interpreted the results and wrote the manuscript.

\section{Conflicts of Interest}

The authors state no conflict of interest. 


\section{References}

1. Feling, R.H.; Buchanan, G.O.; Mincer, T.J.; Kauffman, C.A.; Jensen, P.R.; Fenical, W. Salinosporamide A: A highly cytotoxic proteasome inhibitor from a novel microbial source, a marine bacterium of the new genus Salinospora. Angew. Chem. Int. Ed. 2003, 42, 355-357.

2. Hughes, C.C.; Prieto-Davo, A.; Jensen, P.R.; Fenical, W. The marinopyrroles, antibiotics of an unprecedented structure class from a marine Streptomyces sp. Org. Lett. 2008, 10, 629-631.

3. Fenical, W.; Jensen, P.R.; Palladino, M.A.; Lam, K.S.; Lloyd, G.K.; Potts, B.C. Discovery and development of the anticancer agent salinosporamide A (NPI-0052). Bioorg. Med. Chem. 2009, $17,2175-2180$.

4. Wolfender, J.L.; Marti, G.; Queiroz, E.F. Advances in techniques for profiling crude extracts and for the rapid identification of natural products: dereplication, quality control and metabolomics. Curr. Org. Chem. 2010, 14, 1808-1832.

5. Wang, J.P.; Lin, W.H.; Wray, V.; Lai, D.W.; Proksch, P. Induced production of depsipeptides by co-culturing Fusarium tricinctum and Fusarium begoniae. Tetrahedron Lett. 2013, 54, 2492-2496.

6. Nett, M.; Ikeda, H.; Moore, B.S. Genomic basis for natural product biosynthetic diversity in the actinomycetes. Nat. Prod. Rep. 2009, 26, 1362-1384.

7. Winter, J.M.; Behnken, S.; Hertweck, C. Genomics-inspired discovery of natural products. Curr. Opin. Chem. Biol. 2011, 15, 22-31.

8. Schmitt, E.K.; Moore, C.M.; Krastel, P.; Petersen, F. Natural products as catalysts for innovation: a pharmaceutical industry perspective. Curr. Opin. Chem. Biol. 2011, 15, 497-504.

9. Chiang, Y.M.; Chang, S.L.; Oakley, B.R.; Wang, C.C. Recent advances in awakening silent biosynthetic gene clusters and linking orphan clusters to natural products in microorganisms. Curr. Opin. Chem. Biol. 2011, 15, 137-143.

10. Scherlach, K.; Hertweck, C. Triggering cryptic natural product biosynthesis in microorganisms. Org. Biomol. Chem. 2009, 7, 1753-1760.

11. Schroeckh, V.; Scherlach, K.; Nutzmann, H.W.; Shelest, E.; Schmidt-Heck, W.; Schuemann, J.; Martin, K.; Hertweck, C.; Brakhage, A.A. Intimate bacterial-fungal interaction triggers biosynthesis of archetypal polyketides in Aspergillus nidulans. Proc. Natl. Acad. Sci. USA 2009, 106, 14558-14563.

12. Zuck, K.M.; Shipley, S.; Newman, D.J. Induced production of $N$-formyl alkaloids from Aspergillus fumigatus by co-culture with Streptomyces peucetius. J. Nat. Prod. 2011, 74, 1653-1657.

13. Pettit, R.K. Mixed fermentation for natural product drug discovery. Appl. Microbiol. Biotechnol. 2009, 83, 19-25.

14. Cueto, M.; Jensen, P.R.; Kauffman, C.; Fenical, W.; Lobkovsky, E.; Clardy, J. Pestalone, a new antibiotic produced by a marine fungus in response to bacterial challenge. J. Nat. Prod. 2001, 64, 1444-1446.

15. Oh, D.C.; Jensen, P.R.; Kauffman, C.A.; Fenical, W. Libertellenones A-D: Induction of cytotoxic diterpenoid biosynthesis by marine microbial competition. Bioorg. Med. Chem. 2005, $13,5267-5273$. 
16. Oh, D.-C.; Kauffman, C.A.; Jensen, P.R.; Fenical, W. Induced production of emericellamides A and B from the marine-derived fungus Emericella sp. in competing co-culture. J. Nat. Prod. 2007, $70,515-520$.

17. Lisdiyanti, P.; Otoguro, M.; Ratnakomala, S.; Lestari, Y.; Hastuti, R.D.; Triana, E.; Katsuhiko, A.; Widyastuti, Y. Actinokineospora baliensis sp. nov., Actinokineospora cibodasensis sp. nov. and Actinokineospora cianjurensis sp. nov., isolated from soil and plant litter. Int. J. Syst. Evol. Microbiol. 2010, 60, 2331-2335.

18. Tang, X.; Zhou, Y.; Zhang, J.; Ming, H.; Nie, G.X.; Yang, L.L.; Tang, S.K.; Li, W.J. Actinokineospora soli sp. nov., a thermotolerant actinomycete isolated from soil, and emended description of the genus Actinokineospora. Int. J. Syst. Evol. Microbiol. 2012, 62, 1845-1849.

19. Abdelmohsen, U.R.; Pimentel-Elardo, S.M.; Hanora, A.; Radwan, M.; Abou-El-Ela, S.H.; Ahmed, S.; Hentschel, U. Isolation, phylogenetic analysis and anti-infective activity screening of marine sponge-associated actinomycetes. Mar. Drugs 2010, 8, 399-412.

20. Abdelmohsen, U.R.; Cheng, C.; Viegelmann, C.; Zhang, T.; Grkovic, T.; Quinn, R.J.; Safwat, A.; Hentschel, U.; Edrada-Ebel, R. Dereplication strategies for targeted isolation of new anti-trypanosomal actinosporins A and B from a marine sponge associated-Actinokineospora sp. EG49. Mar. Drugs 2014, 12, 1220-1244.

21. Engelhardt, K.; Degnes, K.F.; Kemmler, M.; Bredholt, H.; Fjaervik, E.; Klinkenberg, G.; Sletta, H.; Ellingsen, T.E.; Zotchev, S.B. Production of a new thiopeptide antibiotic, TP-1161, by a marine Nocardiopsis species. Appl. Environ. Microbiol. 2010, 76, 4969-4976.

22. Selvin, J.; Shanmughapriya, S.; Gandhimathi, R.; Seghal Kiran, G.; Rajeetha Ravji, T.; Natarajaseenivasan, K.; Hema, T.A. Optimization and production of novel antimicrobial agents from sponge associated marine actinomycetes Nocardiopsis dassonvillei MAD08. Appl. Microbiol. Biotechnol. 2009, 83, 435-445.

23. Xu, S.; Yan, L.; Zhang, X.; Wang, C.; Feng, G.; Li, J. Nocardiopsis fildesensis sp. nov., an actinomycete isolated from soil. Int. J. Syst. Evol. Microbiol. 2014, 64, 174-179.

24. Wu, Z.C.; Li, S.; Nam, S.J.; Liu, Z.; Zhang, C. Nocardiamides A and B, two cyclohexapeptides from the marine-derived actinomycete Nocardiopsis sp. Cnx037. J. Nat. Prod. 2013, 76, 694-701.

25. Schneemann, I.; Ohlendorf, B.; Zinecker, H.; Nagel, K.; Wiese, J.; Imhoff, J.F. Nocapyrones A-D, $\gamma$-pyrones from a Nocardiopsis strain isolated from the marine sponge Halichondria panicea. J. Nat. Prod. 2010, 73, 1444-1447.

26. Cho, J.Y.; Williams, P.G.; Kwon, H.C.; Jensen, P.R.; Fenical, W. Lucentamycins A-D, cytotoxic peptides from the marine-derived actinomycete Nocardiopsis lucentensis. J. Nat. Prod. 2007, 70, $1321-1328$.

27. Zhao, X.Q. Genome-based studies of marine microorganisms to maximize the diversity of natural products discovery for medical treatments. Evid. Based Complement. Alternat. Med. 2011, 2011, $1-11$.

28. Rateb, M.E.; Hallyburton, I.; Houssen, W.E.; Bull, A.T.; Goodfellow, M.; Santhanam, R.; Jaspars, M.; Ebel, R. Induction of diverse secondary metabolites in Aspergillus fumigatus by microbial co-culture. RSC Adv. 2013, 3, 14444-14450. 
29. Park, H.B.; Kwon, H.C.; Lee, C.H.; Yang, H.O. Glionitrin A, an antibiotic-antitumor metabolite derived from competitive interaction between abandoned mine microbes. J. Nat. Prod. 2009, 72, 248-252.

30. Bertrand, S.; Schumpp, O.; Bohni, N.; Monod, M.; Gindro, K.; Wolfender, J.L. De novo production of metabolites by fungal co-culture of Trichophyton rubrum and Bionectria ochroleuca. J. Nat. Prod. 2013, 76, 1157-1165.

31. Schmidtz, F.J.; Vanderah, D.J.; Hollenbeak, K.H.; Enwall, C.E.L.; Gopichand, Y.; SenGupta, P.K.; Hossain, M.B.; Van der Helm, D. Metabolites from the marine sponge Tedania ignis. A new atisanediol and several known diketopiperazines. J. Org. Chem. 1983, 48, 3941-3945.

32. Adamczeski, M.; Reed, A.R.; Crews, P. New and known diketopiperazines from the caribbean sponge, Calyx cf. Podatypa. J. Nat. Prod. 1995, 58, 201-208.

33. Jayatilake, G.S.; Thornton, M.P.; Leonard, A.C.; Grimwade, J.E.; Baker, B.J. Metabolites from an antarctic sponge-associated bacterium, Pseudomonas aeruginosa. J. Nat. Prod. 1996, 59, 293-296.

34. Birch, A.J.; Russell, R.A. Studies in relation to biosynthesis-XLIV: Structural elucidations of brevianamides-B, -C, -D and -F. Tetrahedron 1972, 28, 2999-3008.

35. Wen-ju, H.; Xiao-ling, L.; Qiang-zhi, X.; Xiao-yu, L.; Bing-hua, J. Isolation, identification and biological characterization of secondary metabolites produced by a marine Bacillus subtilis. Acad. J. Sec. Mil. Med. Univ. 2008, 29, 1234-1238.

36. Fraga, B.M.; Diaz, C.E.; Quintana, N. Naphthohydroquinones and lignans from the roots of Plocama pendula, a canary island paleoendemism. Biochem. Syst. Ecol. 2010, 38, 784-788.

37. Joshi, B.S.; Kamat, V.N.; Gawad, D.H. Some $\beta$-carboline alkaloids of Ailanthus malabarica DC. Heterocycles 1977, 7, 193-200.

38. Pusecker, K.; Laatsch, H.; Helmke, E.; Weyland, H. Dihydrophencomycin methyl ester, a new phenazine derivative from a marine streptomycete. J. Antibiot. 1997, 50, 479-483.

39. Mateo Alonso, A.; Horcajada, R.; Groombridge, H.J.; Chudasama Née Mandalia, R.; Motevalli, M.; Utley, J.H.; Wyatt, P.B. Synthesis of phenazine derivatives for use as precursors to electrochemically generated bases. Org. Biomol. Chem. 2005, 3, 2832-2841.

40. Barluenga, J.; Aznar, F.; Liz, R.; Cabal, M.-P.; Cano, F.H.; Foces-Foces, C. Oxidative aminomercuration of 2-propyn-1-ols. Stereoselective syntheses and structures ofcis-[1,4]oxazino [3,2-b]-1,4-oxazine derivatives. Chem. Berichte 1986, 119, 887-899.

41. Sheldrake, H.M.; Jamieson, C.; Burton, J.W. The changing faces of halogenated marine natural products: total synthesis of the reported structures of elatenyne and an enyne from Laurencia majuscula. Angew. Chem. Int. Ed. 2006, 45, 7199-7202.

42. Farfán, N.; Santillan, R.L.; Castillo, D.; Cruz, R.; Joseph-Nathan, P.; Daran, J.-C. Fused heterocycles derived from pseudoephedrine and ephedrine. Can. J. Chem. 1992, 70, 2764-2770.

43. Tauer, E.; Grellmann, K.H. Photochemical dehydrogenation, ring contraction, and ring expansion of hydrogenated derivatives of benzoxazino-benzoxazine, quinoxalino-quinoxaline, and bibenzothiazole. Chem. Berichte 1990, 123, 1149-1154.

44. Huber, W.; Koella, J.C. A comparison of three methods of estimating $\mathrm{EC}_{50}$ in studies of drug resistance of malaria parasites. Acta Trop. 1993, 55, 257-261. 
45. Ponte-Sucre, A.; Vicik, R.; Schultheis, M.; Schirmeister, T.; Moll, H. Aziridine-2,3-dicarboxylates, peptidomimetic cysteine protease inhibitors with antileishmanial activity. Antimicrob. Agents Chemother. 2006, 50, 2439-2447.

(C) 2014 by the authors; licensee MDPI, Basel, Switzerland. This article is an open access article distributed under the terms and conditions of the Creative Commons Attribution license (http://creativecommons.org/licenses/by/3.0/). 